\title{
28 Resarach Soure \\ Teaching the Technical Essentials of Direct Ophthalmoscopy Using a Portable Fundus Camera
}

\author{
Weibo Feng \\ Sun Yat-sen University \\ Yuxian Zou \\ Sun Yat-sen University \\ Yonghao Li \\ Sun Yat-sen University \\ Tao Shen \\ Sun Yat-sen University \\ Ya Gao \\ Sun Yat-sen University \\ Lin Lu \\ Sun Yat-sen University \\ Bingqian Liu ( $\sim$ liubq6@mail.sysu.edu.cn ) \\ Sun Yat-sen University
}

\section{Research Article}

Keywords: Direct Ophthalmoscopy, Medical Education, Internship

Posted Date: September 16th, 2021

DOl: https://doi.org/10.21203/rs.3.rs-787963/v1

License: (c) (i) This work is licensed under a Creative Commons Attribution 4.0 International License.

Read Full License 


\section{Abstract}

Aim: A portable fundus camera could allow the third person to observe the technique essentials of direct ophthalmoscopy from a real time screen. This study was designed to compare the proficiency of teaching direct ophthalmoscopy using a portable camera with conventional way in medical students.

Methods: Medical students of fourth year were invited to participate the study. At baseline, the participants were taught fundoscopy with a conventional direct ophthalmoscope shortly. Then they were randomized to be taught the skill of fundoscopy either with a portable fundus camera or with a conventional direct ophthalmoscope as control for two days. Accuracy tests to match a subject's fundus with one of the four photographs after examining an undilated eye using a direct ophthalmoscope were performed at baseline and end point. Accuracy test scores and self-reported confidence were compared between the two groups.

Results: A total of 160 students participated the study, with 79 assigned to the intervention group, and 81 to the control group. All the students finished the study. At baseline, there was no difference in accuracy test score between the two groups. After two-day training session, the accuracy score improved in $26 / 79$ (32.9\%) students of intervention group versus 15/81 (18.5\%) of the control group $(p=0.037)$. At end point, a total of $39 / 79(49.4 \%)$ students in the intervention groups versus $25 / 81(30.9 \%)$ in the control group identified the correct fundus photograph $(p=0.017)$. The confidence levels were significantly higher in the intervention group than the control group.

Conclusions: Teaching direct ophthalmoscope using a portable fundus camera is associated with improved accuracy score and elevated confidence level in medical students when compared with conventional method.

\section{Introduction}

It is well known that fundus examination is important for diagnosis of many life- and vision-threatening diseases. Ophthalmoscopy allows the observer to view the retina for pathologic changes, particularly in patients with retinal diseases such as glaucoma, retinal detachment, diabetic retinopathy, hypertensive retinopathy, increased intracranial pressure, occluded retinal vessels, and a series of maculopathies including macular edema, macular degeneration, macular hole, macular atrophy, epiretinal membrane, et al. Direct ophthalmoscopy is a simple, economic, and efficient technique for examination of the retina. It can be performed through a undilated- or dilated pupil within minutes during the general medical examination.

It is strongly recommended that all medical students and general physicians should be proficient in direct ophthalmoscopy. However, students and doctors find direct ophthalmoscopy a difficult skill to learn and there is a shortage of ophthalmoscopy in clinical practice shown by a lot of documents ${ }^{1-3}$. In the TOTeMS study, students favored fundus photographs instead of direct ophthalmoscope ${ }^{4}$, further suggesting the skill of ophthalmoscopy was not easy to learn. 
Enough time and practice are important to master the teaching skill of direct ophthalmoscopy for medical students. But nowadays shorter duration is provided for teaching this basic technique in medical curricula due to time constraints. Several reports were designed to improve the learning of ophthalmoscopy. For example, teaching the mechanics of direct ophthalmoscopy with simulators or models was found useful ${ }^{5-11}$. A portable fundus camera could allow the third person to observe the technique essentials of direct ophthalmoscopy from a real time screen. This study was designed to compare the proficiency of teaching direct ophthalmoscopy using a portable camera with conventional way in medical students during ophthalmic clerkship.

\section{Methods}

\section{Study population}

This was a prospective, randomized study. Participants were all from fourth-year medical students at Sun Yat-sen University. The study was performed during the period of ophthalmic clerkship. Informed consent was signed by all the participants, who confirmed never received training of direct ophthalmoscopy until being enrolled in this study.

\section{Overview of protocol}

The protocol of this study is shown in Figure 1. The consented participants were given lecture of funds examination, and provided illustration of techniques of direct ophthalmoscopy for 10 minutes. Then, they practiced ophthalmoscopy for two hours, while the tutor took fundus photo of each participant. After twohour practice, baseline accuracy test was performed. Then, the participants were randomized into intervention or control group. During the next two-day practice in the intervention group, the key skills of ophthalmoscopy were illustrated with a portable fundus camera which displayed the subject's fundus on real time, while in the control group, the key skills of ophthalmoscopy were illustrated in a conventional way. Figure $2 \mathrm{~A}$ shows the tutor is showing technical essentials of fundus ophthalmoscopy with a portable fundus camera in the intervention group. After the two-day practice with direct ophthalmoscopy, end point accuracy test was performed and final survey questionnaires were collected.

\section{Accuracy test}

Matching fundus photographs with ophthalmoscopy observation was an objective strategy for evaluating the accuracy of direct ophthalmoscopy ${ }^{12-14}$. Our accurate test was performed in a similar way. In brief, assessment of direct ophthalmoscopy skills was done using four fundus photographs, taken from enrolled participants by tutor. Figure $2 \mathrm{~B}$ shows an array of fundus photographs taken with a portable camera from the participants. Four fundus photographs were shown on computer screen, one of which was identical to fundus of the subject. The students were asked to identify the correct image after examining a subject's undilated eye. Three minutes were allotted for each student. Both the baseline and end point accuracy tests were performed by masked examiners. 


\section{Final survey}

A final survey was administered after all training and testing sequences were complete. It is asked in the control group what is the main frustration during the learning of direct ophthalmoscopy. In the intervention group the survey asked which illustration methods students preferred for understanding the key skill of direct ophthalmoscope.

\section{Results}

Of 432 fourth year medical students in the medical school of Sun Yat-sen University, 160 consented to participate in the study and all the participants completed required test and final survey. 89 of the 160 were women and the median age was 22 years old. 79 students were assigned to intervention group, and 81 students were assigned to control group.

\section{Accuracy test}

Table 1 shows the changes of accuracy tests from baseline to end point of the two groups. At baseline, the two groups showed no difference in accuracy test performance. 24 of the 79 students in the intervention group, and 21 of the 81 students in the control group, identified the correct fundus photograph of subject in the accuracy test $(p=0.531)$. At the end point, accuracy improvement in ophthalmoscopy was observed in both groups after the two-day training program, with 26/79 students in the intervention group versus $15 / 81$ students in the control group ( $p=0.037)$. At end point, a total of $39 / 79$ in the intervention groups vs $25 / 81$ in the control group identified the correct fundus photograph $(p=0.017)$. Eleven students from each group showed worse accuracy score after training due to unknown reason. Figure 3 shows results of accuracy test of direct ophthalmoscopy at the baseline and end point in the intervention and control groups.

Table 1. Changes of ophthalmoscopy performance from the baseline to the end point in the two groups.

\begin{tabular}{|c|c|c|c|c|}
\hline & Better* & No change & Worse & Better means the photography \\
\hline Intervention $(\mathrm{n}=79)$ & $26(32.9 \%)$ & $42(53.2 \%)$ & $11(13.9 \%)$ & $\begin{array}{l}\text { identification was wrong at baseline, } \\
\text { and correct at end point. Worse means }\end{array}$ \\
\hline Control $(n=81)$ & $15(18.5 \%)$ & $55(67.9 \%)$ & $11(13.4 \%)$ & the photography identification was \\
\hline
\end{tabular}

${ }^{*} \mathrm{p}=0.037$

\section{Confidence levels}

At baseline, the two groups showed no difference in self-reported confidence levels. After two-day training, confidence levels were elevated significantly in the intervention group but showed no change in the control group. Figure 4 shows the self-reported confidence levels at the baseline and end point. 


\section{Discussion}

Many studies reported the underuse of direct ophthalmoscopy, probably because of the techniques are difficult to learn during the limited duration of training. Clerkship is possibly the only chances for medical students to learn knowledge of ophthalmology, but the period is very short for students to learn the techniques of ophthalmoscopy.

There are many ways to illustration of technical essentials of direct ophthalmoscopy. Using a traditional way, many students reported frustrated and cannot learn the skill of ophthalmoscopy. Some reported "Hard to understand what is the teacher illustrating, can't see what is the teacher looking, don't know how to use the ophthalmoscope". Through a smartphone ophthalmoscope or a simultaneous colour television transmission, observers were able to visualize the fundus easier ${ }^{15,16}$. In that way, the students could see what is the teacher looking, but don't know how to use the ophthalmoscope, that is to say, the technical essentials of ophthalmoscopy might still remain frustrating for medical students. From the results of our control group, we found the conventional way of illustrating ophthalmoscopy was associated with lower level of understanding of this technique, lower interest to fundus examination, lower accuracy test score improvement, and lower self-reported confidence.

Using a portable fundus camera to illustrate the technique essentials of direct ophthalmoscopy has several benefits: it allows students and trainers to share the same view of the fundus on real time; learning with such a device is associated with greater confidence and competence in students using the direct ophthalmoscope. With a portable fundus camera, the students found it easy to understand the technical essentials including the "importance of subject's cooperation, right direction of looking through pupil, and adjusting the focus of ophthalmoscope" by watching the fundus imaging on the camera screen. Using a portable fundus to illustrate the ophthalmoscopy was associated with higher level of understanding of this technique, higher interest to fundus examination, higher accuracy score improvement, and higher confidence.

Limitations of this study: In this study, the accuracy score examiners were masked to the study grouping, but it was not possible to mask the participants or the tutors; The same tutor performed illustrating ophthalmoscopy for both groups, with similar amounts of technique essentials in order to limit the potential bias introduced by different illustration methods; the assessment of accuracy is judged using an array of fundus photographs, it did not absolutely mean that the students who got positive score had identified the fundus with matched photographs, especially at baseline. Equal amounts of students (11) showed worse accuracy score after training in the two groups. Evaluation of ophthalmoscopy accuracy by incorporating a teaching camera into the direct ophthalmoscope which allows the tutor to share the same view of the fundus simultaneously might be even more objective.

\section{Conclusions}


In conclusion, teaching technical essentials of direct ophthalmoscope using a portable fundus camera is associated with improved accuracy score and elevated confidence level in medical students when compared with conventional method.

\section{Abbreviations}

Not applicable

\section{Declarations}

\section{Ethics approval and consent to participate}

It was in adherence the tenets of the Declaration of Helsinki, and was approved by the Zhongshan Ophthalmic Center Institutional Review Board. Human or animal experiment was not involved in this research, and ethics approval and consent to participate were not required.

\section{Consent for publication}

Not applicable

\section{Availability of data and material}

All data generated or analyzed during this study are included in this published article.

\section{Competing interests}

The authors declare that they have no competing interests

\section{Funding}

This study is funded by college educational reform program, Sun Yat-sen University

\section{Authors' contributions}

WB.F, YX.Z, BQ,L were for study design. YH.L and L.L provided the equipment. T.S was for statistic analysis. Y.G was for student organization. All authors habe read and approved the manuscript.

\section{Acknowledgements}

The authors thank Pro. Yehong Zhuo, Pro. Danying Zheng for their kindly assistance as counselors in this study.

\section{References}


1. Gupta RR, Lam WC. Medical students' self-confidence in performing direct ophthalmoscopy in clinical training. Can J Ophthalmol 2006;41(2):169-74.

2. Mackay DD, Garza PS, Bruce BB, et al. The demise of direct ophthalmoscopy: A modern clinical challenge. Neurol Clin Pract 2015;5(2):150-7.

3. Benbassat J, Polak BC, Javitt JC. Objectives of teaching direct ophthalmoscopy to medical students. Acta Ophthalmol 2012;90(6):503-7.

4. Kelly LP, Garza PS, Bruce BB, et al. Teaching ophthalmoscopy to medical students (the TOTeMS study). Am J Ophthalmol 2013;156(5):1056-61 e10.

5. Chung KD, Watzke RC. A simple device for teaching direct ophthalmoscopy to primary care practitioners. Am J Ophthalmol 2004;138(3):501-2.

6. Akaishi Y, Otaki J, Takahashi O, et al. Validity of direct ophthalmoscopy skill evaluation with ocular fundus examination simulators. Can J Ophthalmol 2014;49(4):377-81.

7. Hoeg TB, Sheth BP, Bragg DS, Kivlin JD. Evaluation of a tool to teach medical students direct ophthalmoscopy. WMJ 2009;108(1):24-6.

8. Kouzmitcheva E, Grover SA, Berenbaum T, et al. Evaluation of an Ophthalmoscopy Simulator to Teach Funduscopy Skills to Pediatric Residents. Can J Neurol Sci 2018;45(3):320-4.

9. Westermeyer HD, Druley GE, Royal KD, Mowat FM. Use of a Versatile, Inexpensive Ophthalmoscopy Teaching Model in Veterinary Medical Student Education Increases Ophthalmoscopy Proficiency. J Vet Med Educ 2019:1-5.

10. Swanson S, Ku T, Chou C. Assessment of direct ophthalmoscopy teaching using plastic canisters. Med Educ 2011;45(5):520-1.

11. Yusuf $I H$, Ridyard E, Fung THM, et al. Integrating retinal simulation with a peer-assessed group OSCE format to teach direct ophthalmoscopy. Can J Ophthalmol 2017;52(4):392-7.

12. Krohn J, Kjersem B, Hovding G. Matching fundus photographs of classmates. An informal competition to promote learning and practice of direct ophthalmoscopy among medical students. $J$ Vis Commun Med 2014;37(1-2):13-8.

13. Kwok J, Liao W, Baxter S. Evaluation of an online peer fundus photograph matching program in teaching direct ophthalmoscopy to medical students. Can J Ophthalmol 2017;52(5):441-6.

14. Milani BY, Majdi M, Green W, et al. The use of peer optic nerve photographs for teaching direct ophthalmoscopy. Ophthalmology 2013;120(4):761-5.

15. Kim Y, Chao DL. Comparison of smartphone ophthalmoscopy vs conventional direct ophthalmoscopy as a teaching tool for medical students: the COSMOS study. Clin Ophthalmol 2019;13:391-401.

16. Scherfig E, Edmund J, Northeved A. Direct ophthalmoscopy with simultaneous colour television transmission. Acta Ophthalmol (Copenh) 1979;57(4):649-52.

\section{Figures}


Consented participants: $4^{\text {th }}$ year medical students $(n=160)$

Tutor demonstrating direct ophthalmoscopy for $10 \mathrm{~min}$

Participants practice with each other for 2 hours.

(Tutor takes fundus photos of participants)

\section{Baseline assessment and randomization of participants}

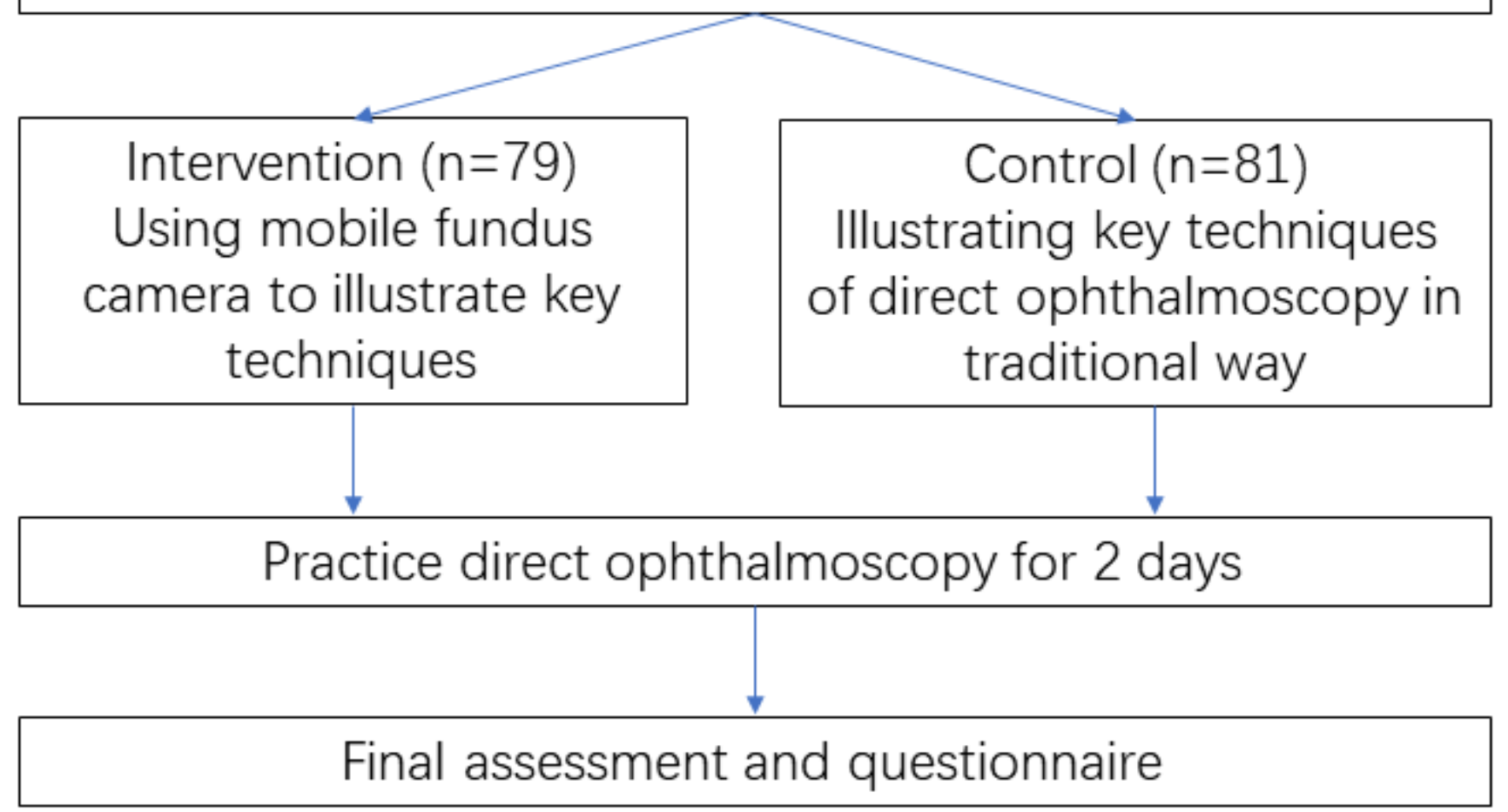

Figure 1

Protocol of the study. 

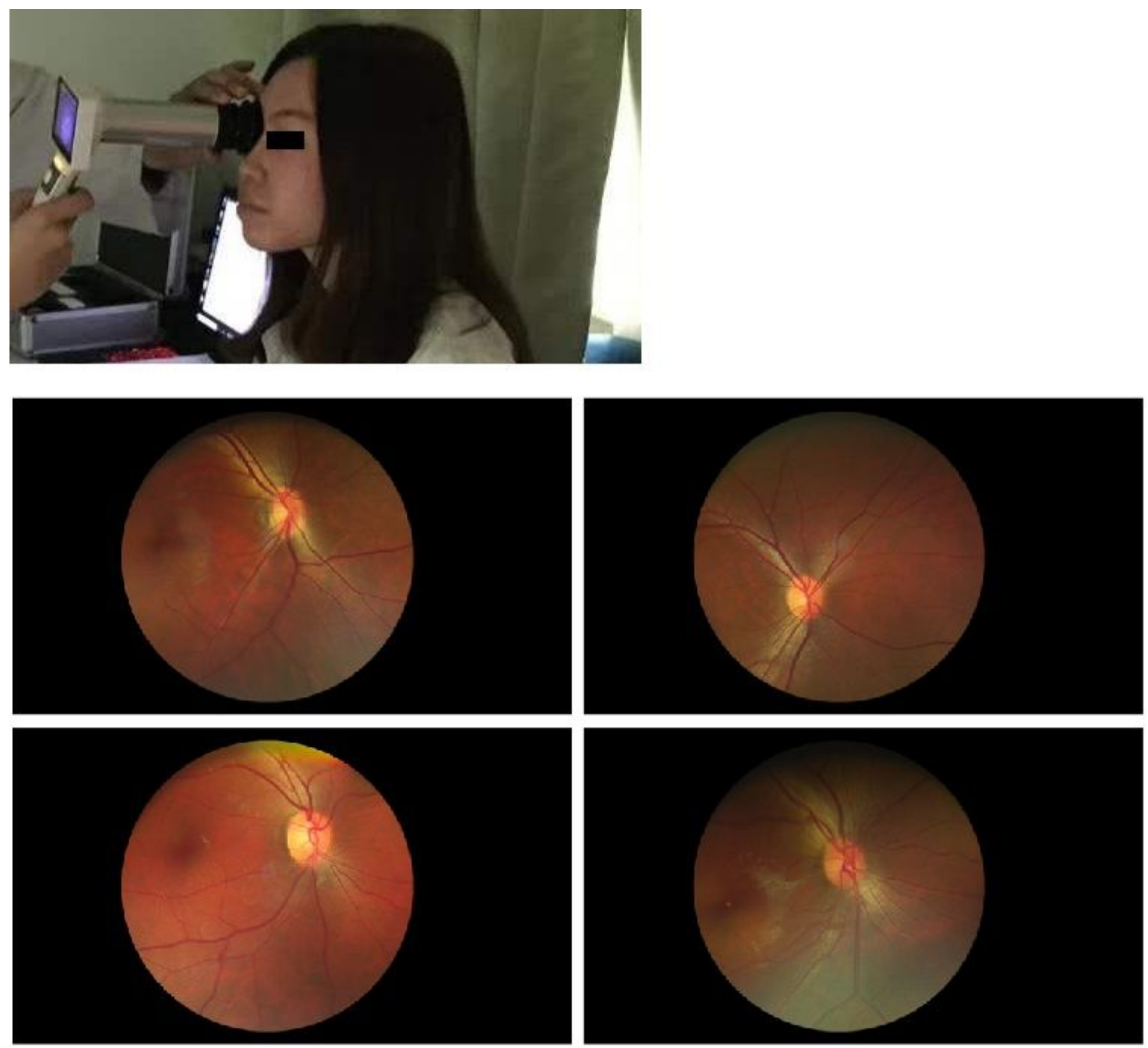

Figure 2

A. The tutor is showing technical essentials of fundus ophthalmoscopy with a portable fundus camera in the intervention group. B. Fundus photographs taken with a portable camera from the participants. For accuracy test, four fundus photographs were shown to each student, one of which was identical to fundus of the subject. The students was asked to identify the correct image after examining a subject's undilated eye. 


$$
P=0.013 \quad P=0.017
$$
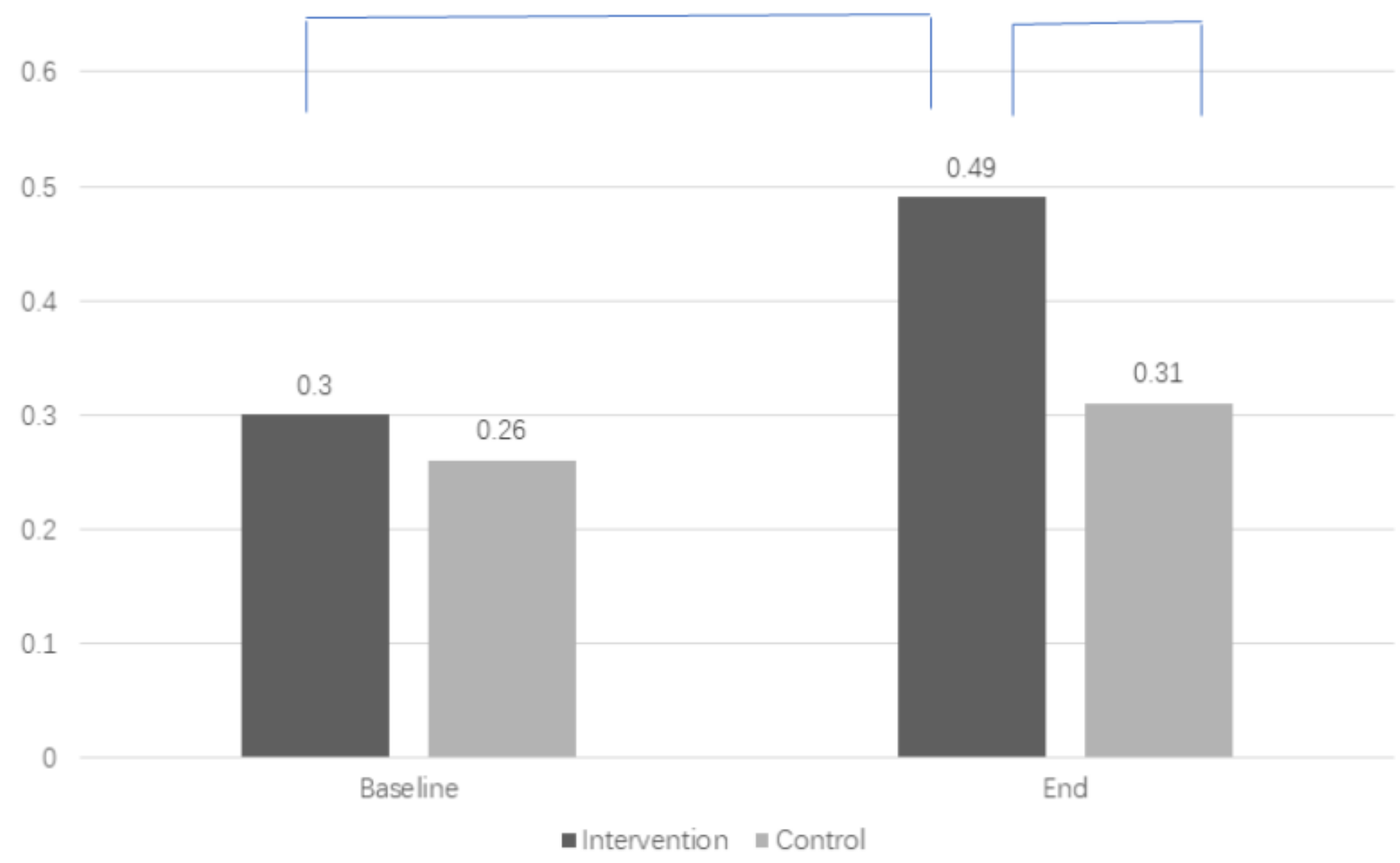

Figure 3

Accuracy test of direct ophthalmoscopy at the baseline and end in the intervention and control groups. 


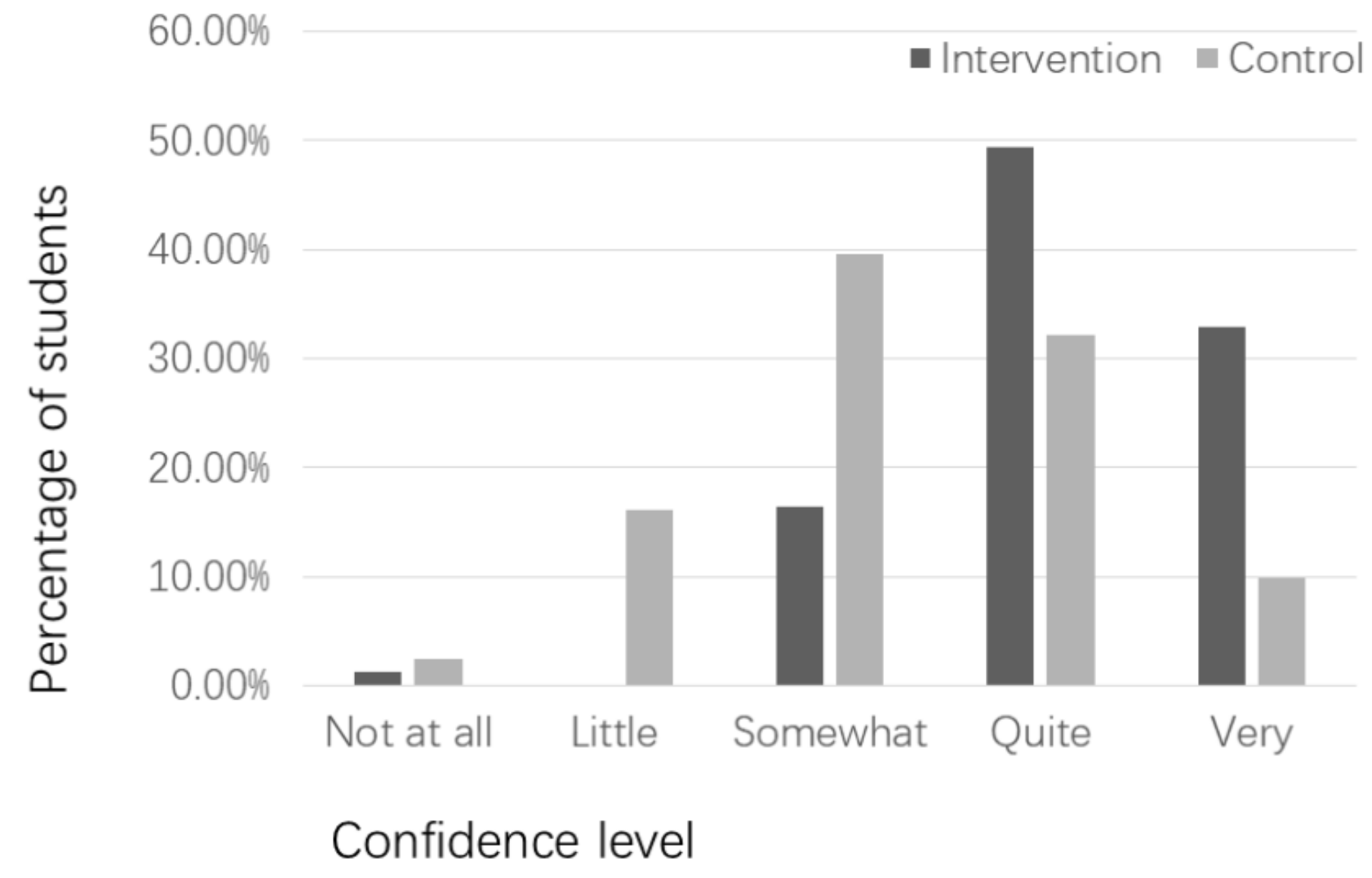

Figure 4

Self-reported confidence levels of direct ophthalmoscopy in the intervention and control groups at the end point of the study. 